\section{Identificação de grupos prioritários para a vacinação contra COVID-19 no Brasil}

\author{
Identification of priority groups for COVID-19 \\ vaccination in Brazil
}

\section{Identificación de grupos prioritarios para la vacunación contra la COVID-19 en Brasil}

\author{
Raquel Martins Lana 1 \\ Lais Picinini Freitas 1 \\ Cláudia Torres Codeço 1 \\ Antônio Guilherme Pacheco 1 \\ Luiz Max Fagundes de Carvalho 2 \\ Daniel Antunes Maciel Villela 1 \\ Flávio Codeço Coelho 2,3 \\ Oswaldo Gonçalves Cruz 1 \\ Roberta Pereira Niquini 4 \\ Victor Bertollo Gomes Porto 5 \\ Caroline Gava 5 \\ Marcelo Ferreira da Costa Gomes 1 \\ Leonardo Soares Bastos 1
}

doi: 10.1590/0102-311X00049821

\section{Resumo}

Em um contexto de transmissão comunitária e escassez de vacinas, a vacinação contra a COVID-19 deve focar na redução direta da morbidade e da mortalidade causadas pela doença. Portanto, é fundamental a definição de grupos prioritários para a vacinação pelo Programa Nacional de Imunizações (PNI), baseada no risco de hospitalização e óbito pela doença. Para tal, calculamos o sobrerrisco por sexo, faixa etária e comorbidades por meio dos registros de hospitalização e óbito por síndrome respiratória aguda grave com confirmação de COVID-19 (SRAG-COVID) em todo o Brasil nos primeiros seis meses de epidemia. Apresentaram maior sobrerrisco pessoas do sexo masculino (hospitalização $=1,1$ e óbito $=1,2$ ), pessoas acima de 45 anos para hospitalização $\left(\mathrm{SR}_{\mathrm{fe}}\right.$ variando de 1,1 a 8,5) e pessoas acima de 55 anos para óbitos $\left(\mathrm{SR}_{\mathrm{fe}}\right.$ variando de 1,5 a 18,3). Nos grupos de comorbidades, doença renal crônica, diabetes mellitus, doença cardiovascular e pneumopatia crônica conferiram sobrerrisco, enquanto para asma não houve evidência. Ter doença renal crônica ou diabetes mellitus e 60 anos ou mais mostrou-se um fator ainda mais forte, alcançando sobrerrisco de óbito 14 e 10 vezes maior do que na população geral, respectivamente. Para todas as comorbidades, houve um sobrerrisco mais alto em idades maiores, com um gradiente de diminuição em faixas mais altas. Esse padrão se inverteu quando consideramos o sobrerrisco em relação à população geral, tanto para hospitalização quanto para óbito. O presente estudo forneceu evidências a respeito do sobrerrisco de hospitalização e óbito por SRAG-COVID, auxiliando na definição de grupos prioritários para a vacinação contra a COVID-19.

COVID-19; Síndrome Respiratória Aguda Grave; Monitoramento

Epidemiológico; Comorbidade

\section{Correspondência}

R. M. Lana

Programa de Computação Científica, Fundação Oswaldo Cruz. Av. Brasil 4365, Rio de Janeiro, RJ 21040-900, Brasil. raquelmlana@gmail.com

1 Programa de Computação Científica, Fundação Oswaldo Cruz, Rio de Janeiro, Brasil.

2 Fundação Getúlio Vargas, Rio de Janeiro, Brasil.

3 Institute of Global Health, University of Geneva, Geneva, Switzerland.

4 Instituto Federal de Educação, Ciência e Tecnologia do Rio de Janeiro, Rio de Janeiro, Brasil.

5 Coordenação-geral do Programa Nacional de Imunizações, Ministério da Saúde, Brasília, Brasil. 


\section{Introdução}

O primeiro caso de COVID-19 no Brasil foi oficialmente confirmado no dia 26 de fevereiro de 2020, no Estado de São Paulo. Em pouco mais de um mês, todas as 27 Unidades Federativas (UF) do país já registravam dez ou mais casos da doença. O vírus se espalhou seguindo o fluxo aéreo do país, chegando primeiro nas capitais mais populosas e conectadas a São Paulo e Rio de Janeiro, depois para as cidades das regiões metropolitanas, e gradativamente para o interior 1,2. Nos primeiros 6 meses de pandemia no Brasil (até o dia 22 de agosto de 2020), de acordo com o Painel Coronavírus (https:// covid.saude.gov.br/), já haviam sido registrados oficialmente 5.323 .630 casos e 155.900 óbitos confirmados de COVID-19 no país. Tais registros incluem casos (assintomáticos, leves e graves) e óbitos pelo SARS-CoV-2 confirmados por diferentes exames laboratoriais (RT-PCR, testes rápidos, exames sorológicos), além de eventuais diagnósticos clínicos por imagem 3.

Desde o início da pandemia de COVID-19, houve um grande movimento para disponibilização dos dados e informações que pudessem ajudar em planos de contingência, estratégias de mitigação, dentre outros. Além disso, um grande movimento global reuniu esforços de diversos grupos de pesquisa, instituições e países, na busca por vacinas contra a doença ${ }^{4}$. Em 23 de fevereiro de 2021, mais de 250 vacinas - sendo 182 em desenvolvimento pré-clínico, 73 em desenvolvimento clínico e, dessas, 16 em fase III - estavam sendo testadas 5. Desse montante, cinco já estavam aprovadas para uso em alguns países 6 sendo duas vacinas aprovadas para uso emergencial no Brasil 7 e uma aprovada com registro definitivo ${ }^{8}$. Era de conhecimento anterior à liberação dessas vacinas que um plano de distribuição das doses por grupos prioritários seria necessário, devido à baixa disponibilidade de doses para a demanda mundial 9 . Especificamente para o Brasil, país de dimensão continental com cerca de 210 milhões de habitantes, a definição de grupos prioritários é condição essencial para qualquer campanha dessa magnitude.

O Programa Nacional de Imunizações (PNI), reconhecido mundialmente por sua capacidade de atender a um país com dimensões continentais e de grande diversidade socioeconômica, com um calendário vasto de vacinas, é um importante braço do Sistema Único de Saúde (SUS) (Departamento de Informática do SUS. Sistema de Informações do Programa Nacional de Imunizações. http://sipni.datasus.gov.br/si-pni-web/faces/apresentacaoSite.jsf, acessado em 16/Fev/2021), e o responsável pelo Plano Nacional de Operacionalização da Vacinação contra a COVID-19 10. Dentre as atividades do PNI está a campanha anual para vacinação contra influenza (H1N1), que, de forma similar à COVID-19, é organizada em grupos prioritários (http://sipni.datasus.gov.br/si-pni-web/ faces/apresentacaoSite.jsf).

De acordo com a OMS, a definição de grupos prioritários para vacinação contra COVID-19 num contexto de transmissão comunitária deve ter como foco em seu estágio inicial, a redução direta da morbidade e da mortalidade, além da manutenção dos serviços essenciais, e honrar o princípio da reciprocidade (proteger aqueles indivíduos com alta exposição ao vírus por serviço à comunidade) 9 .

Portanto, o principal objetivo das presentes análises foi definir grupos de risco para hospitalização e óbito por COVID-19. Essas análises auxiliaram na definição dos grupos prioritários do plano nacional de vacinação contra a COVID-19 elaborado pelo PNI para o primeiro estágio da campanha. Foi realizado um estudo por meio de análises descritivas de hospitalizações e óbitos por síndrome respiratória aguda grave associada à COVID-19 (SRAG-COVID) notificados nos primeiros seis meses de pandemia no Brasil. Esse estudo é uma atualização e extensão de análises previamente publicadas em Niquini et al. 11, especialmente pelo uso de indicadores de sobrerrisco para a comparação de grupos.

\section{Métodos}

\section{Dados}

Dados de hospitalizações e óbitos por SRAG-COVID foram obtidos do SIVEP-Gripe (https://sivepgripe.saude.gov.br/sivepgripe). O SIVEP-Gripe é o sistema oficial para registro de hospitalizações e óbitos por Síndrome Respiratória Aguda Grave (SRAG) em todo o território nacional. Como parte do protocolo de notificação, todo caso identificado como SRAG tem amostra biológica coletada para 
posterior análise laboratorial. Desde março de 2020, as amostras são testadas também para o vírus SARS-CoV-2. Foram incluídos no estudo somente os casos com registro de confirmação por RT-PCR, exame considerado padrão-ouro para o diagnóstico laboratorial da infecção pelo SARS-CoV-2.

Considerou-se os registros com data de primeiros sintomas até a 35a Semana Epidemiológica (SE) de 2020 (29 de agosto de 2020), de forma a evitar perda de informação em razão dos atrasos de digitação e de atualização do diagnóstico laboratorial. As variáveis do SIVEP-Gripe consideradas para análise de fatores de risco foram: faixa etária (agregadas de 5 em 5 anos, de 0 a 4 anos até 90 anos ou mais), sexo, e presença de comorbidades (diabetes mellitus, doença renal crônica, asma, outras pneumopatias crônicas e doença cardiovascular [DCV] crônica). Informações sobre hipertensão arterial sistêmica (HAS) foram obtidas por meio de buscas de texto no campo aberto que descreve "outras comorbidades". Para a busca de texto foram utilizadas as strings "HAS" ou "H.A.S." ou "HIPERTE” com o auxílio do pacote strin$g r$ (https://CRAN.R-project.org/package=stringr), disponível para o ambiente R (http://www.r-pro ject.org). É importante pontuar que a exclusão da variável obesidade das análises, mesmo essa sendo um importante fator de risco para COVID-19 12,13, se deu pelas falhas no preenchimento da informação (assim como outras comorbidades) e pela dificuldade de se definir obesidade no momento do preenchimento pelo responsável pela notificação. Para avaliação da obesidade é necessário o índice de massa corporal (IMC). Nos dados de notificação, não apenas a variável obesidade é mal preenchida, como também observa-se volume importante de ausência de preenchimento do campo IMC.

Dados demográficos da população brasileira em 2020 por faixa etária e sexo foram obtidos de projeções realizadas pelo Instituto Brasileiro de Geografia e Estatística (IBGE) 14.

Dados de prevalência de comorbidades na população geral e estratificado por faixa etária para o Brasil foram obtidas da Pesquisa Nacional de Saúde (PNS), conduzida em 2013 15,16. As morbidades consideradas foram diabetes mellitus, insuficiência renal crônica (IRC), asma (ou bronquite asmática), doenças no pulmão (tais como enfisema pulmonar, bronquite crônica ou doença pulmonar obstrutiva crônica - DPOC) e DCV.

As DCV, conforme definidas pela PNS, incluem HAS e doenças do coração (tais como infarto, angina, insuficiência cardíaca e outras). Para compatibilizar com essa definição, foi criada uma variável composta no SIVEP-Gripe, DCV, que agrega HAS e DCV. A estratificação etária para comorbidades considerou três faixas, 18-39 anos, 40-59 anos e 60 anos ou mais. Não foi possível calcular a prevalência em idades menores, pois a PNS contempla em sua amostra apenas pessoas de 18 anos ou mais 15,16. Foi feita a estimativa do número de pessoas portadoras de cada comorbidade em 2020, assumindo a prevalência mensurada pela PNS em 2013 15,16. A projeção da população por faixa etária está agregada de 5 em 5 anos como citado acima, portanto, para a faixa etária de 18-39 anos, somou-se os valores projetados para as faixas etárias de 20-24, 25-29, 30-34 e 35-39 anos, e para as idades 18 e 19 anos foi usada a proporção de dois quintos da projeção para a faixa etária de 15-19 anos do IBGE.

\section{Análise de dados}

\section{- Taxa de incidência}

Foram calculadas as taxas de incidência de hospitalizações e óbitos por 1.000 habitantes para SRAG-COVID por sexo, faixa etária, comorbidade e comorbidade por faixa etária. A taxa de incidência no período consiste na razão entre o total de hospitalizações (ou óbitos) por SRAG-COVID no grupo de interesse até a SE 35 em todo o país, dividido pela respectiva projeção da população do grupo em 2020 multiplicado por 1.000 .

\section{- Cálculo do sobrerrisco}

Um indicador comumente empregado na comparação de risco entre grupos é o risco relativo. Contudo, esse indicador não é calculável com os dados disponíveis, uma vez que os grupos em comparação não consistem em pessoas que estavam ou não expostas ao risco sendo estudado. Portanto, optamos por usar o termo sobrerrisco, que é a razão entre a taxa de hospitalização ou óbito num grupo específico dividido pela taxa do mesmo evento na população geral. 
Formalmente, o sobrerrisco $(S R)$ de um determinado grupo $i, S R_{i}$, foi definido como a razão entre a taxa de incidência de hospitalização ou óbitos no grupo $i$ e a taxa de incidência de hospitalização ou óbito na população geral. Seja $y_{i}$ o número de hospitalizações (ou óbitos) no grupo de interesse $i$, e $n_{i}$ a população de referência para o grupo. Seja também $Y$ o total de hospitalizações (ou óbitos) na população geral e seja $N$ o tamanho dessa população. O modelo estatístico para cálculo do sobrerrisco assume que o número de hospitalizações (ou óbitos) por SRAG-COVID segue uma distribuição Binomial, tanto na população geral, quanto nos grupos de interesse. Portanto temos para cada grupo $i$,

$$
y_{i} \sim \operatorname{Binomial}\left(n_{i}, \theta_{i}\right), i=1,2, \ldots J
$$

em que $\theta_{i}$ é a taxa de incidência de hospitalização (ou óbitos) no grupo $i$, e $J$ é o total de grupos.

Para a população geral, assumimos que

$$
Y \sim \operatorname{Binomial}\left(N, \theta_{0}\right) \text {, }
$$

em que $\theta_{0}$ é a taxa de incidência de hospitalização (ou óbitos) na população geral. E desta forma, o sobrerrisco do grupo $i$ é dado por

$$
S R_{i}=\frac{\theta_{i}}{\theta_{0}}
$$

É importante notar que se os grupos consistem em uma partição da população, ou seja $Y=\Sigma y_{i}$ então a distribuição de definida acima é uma aproximação. Para grandes contagens, o erro de aproximação é pequeno.

Para exemplificar o sobrerrisco, considere um cenário hipotético no qual em um determinado grupo com 100 mil pessoas, são registrados 100 óbitos. Suponha que na população geral há $102 \mathrm{mil}$ óbitos em 210 milhões de habitantes. Nesse caso, o $S R_{i}$ é calculado como: (100/100mil)/(102 mil/210 milhões) levando a um $S R_{i}$ aproximado de 2,1. Ou seja, a taxa de incidência do grupo $i$ é cerca de duas vezes a taxa de incidência na população geral.

Para medir a incerteza das estimativas de $S R_{i}$, sob a ótica bayesiana, atribuímos distribuições a priori uniformes para $\theta_{i}$ e $\theta_{0}$, de tal forma que a distribuição a posteriori para cada parâmetro fosse uma distribuição Beta com parâmetros conhecidos. Amostras da distribuição a posteriori de $S R_{i}$ são obtidas via simulação de Monte Carlo com amostragem de tamanho 100.000 da distribuição de probabilidade do $S R$ de cada grupo $i$. Esta amostra permite obter o intervalo de $95 \%$ de credibilidade (IC95\%) para o $S R_{i}$, que é importante para avaliar se o sobrerrisco é significativo, ou seja, quando o limite inferior do intervalo de credibilidade é maior do que o valor 1 . Aplicando esse método ao exemplo hipotético acima, obtém-se um IC95\% de 1,7-2,5.

De forma análoga, define-se o sobrerrisco estratificado (SRE), para calcular o sobrerrisco de uma comorbidade em um estrato específico, por exemplo, um grupo etário. As hospitalizações (ou óbitos) ficam restritas a uma faixa etária específica, e o sobrerrisco estratificado para o grupo $i$ tem como denominador a taxa de incidência para a faixa etária $(f e)$ em questão, ou seja,

$$
S R_{i}(f e)=\frac{\theta_{i, f e}}{\theta_{0, f e}}
$$

em que $\theta_{i, f e}$ e $\theta_{0, f e}$ representam a taxa incidência do grupo $i$ e da população geral para a faixa etária de interesse. A distribuição do $S R E_{i}(f e)$ para o grupo $i$ é derivada de forma análoga à distribuição do sobrerrisco para o grupo $i$.

Para medir a incerteza das estimativas do $S R E$, assume-se que o número de hospitalizações (ou óbitos) por SRAG-COVID também segue uma distribuição Binomial para grupos etários ou grupos com comorbidades.

Dessa forma, o $S R_{i}$ estimado para comorbidade $c$ e faixa etária $f e$, chamado de $S R_{c, f e}$, usa a taxa de incidência de hospitalizações ou óbitos em indivíduos com uma determinada comorbidade e faixa etária como numerador, e os compara com a taxa de incidência da população geral (denominador). $\mathrm{O}$ $S R E_{c, f e}(f e)$ usa o mesmo numerador de $S R_{c, f e}$, mas o denominador é a taxa de incidência na população de faixa etária $f e$. Portanto, a interpretação do $S R E_{c, f e}(f e)$ diz respeito ao sobrerrisco associado a uma 
comorbidade específica, dentro de uma faixa etária específica, ao passo que o $S R_{c, f e}$ leva em conta, além do efeito da comorbidade, o efeito conjunto da própria faixa etária.

\section{Resultados}

No Brasil, o total de hospitalizações com diagnóstico positivo para SARS-CoV-2 por RT-PCR com data de primeiros sintomas até a SE 35 foi de 292.089. Nesse mesmo período, foram registrados 102.562 óbitos em decorrência da SRAG-COVID. Esses valores correspondem a uma taxa de incidência de 1,4 hospitalizações e 0,5 óbitos por 1.000 habitantes, no período.

As taxas de incidência de hospitalizações (1,5 por 1.000 habitantes) e óbitos ( 0,6 por 1.000 habitantes) para o sexo masculino foram maiores do que para o sexo feminino. Isso se traduz no sobrerrisco de hospitalização aumentado para o sexo masculino de 1,115 (IC95\%: 1,108; 1,122) e reduzido para o feminino, $S R_{s}=0,836(0,831 ; 0,842)$, respectivamente. Para óbitos, a diferença entre os sexos é ainda maior, $\operatorname{com} S R_{s}=1,173(1,161 ; 1,184)$ para o sexo masculino e $S R_{s}=0,831($ IC95\%: 0,822; 0,841) para o feminino.

A Figura 1a apresenta as taxas de incidência de hospitalizações e óbitos de SRAG-COVID por 1.000 habitantes por faixa etária. A Figura $1 \mathrm{~b}$ apresenta o $S R_{f e}$ por faixa etária, que tem como referência a taxa de incidência de hospitalizações ou óbitos (Figura 1a) por 1.000 habitantes na população total. Os valores encontrados indicam proteção relativa nas faixas etárias de 0 a 4 anos $\left(S R_{f e}=0,150\right)$, até 40-44 anos $\left(S R_{f e}=0,904\right)$, com $S R_{f e}$ para hospitalização atingindo valor mínimo entre 10 e 14 anos $\left(S R_{f e}=0,033\right)$. A partir da faixa etária de $45-49$ anos $\left(S R_{f e}=1,133\right)$, há um aumento gradual do sobrerrisco de hospitalização, atingindo $S R_{f e}=8,466$ dentre aqueles com 90 anos ou mais. Em relação aos óbitos, observa-se proteção relativa forte daqueles com até 24 anos de idade $\left(S R_{f e}<0,050\right.$ para todas as faixas), mas que perde força a partir dos 25-29 anos $\left(S R_{f e}=0,144\right)$ até os 50-54 anos $\left(S R_{f e}=\right.$ $0,948)$. A partir da faixa etária de 55-59 anos, com $S R_{f e}=1,452$, observa-se aumento gradual até atingir $S R_{f e}=18,330$ naqueles com 90 anos ou mais.

Na Tabela 1, apresentamos a prevalência e o total de pessoas com comorbidades por faixa etária no Brasil estimada pela PNS 12. Observa-se que a prevalência das comorbidades, com exceção da asma, aumenta com a idade. As DCV se destacam pela prevalência mais alta em relação às outras comorbidades em todas as faixas etárias. Na mesma tabela, observa-se também o número absoluto e as taxas de incidências de hospitalizações e óbitos por SRAG-COVID por mil habitantes, estratificadas por comorbidade e faixa etária.

A Tabela 2 mostra as estimativas de sobrerrisco de hospitalização e óbito estratificadas por comorbidade e faixa etária. Dois indicadores são apresentados, diferenciados pelo denominador utilizado. $\mathrm{O} S R_{c, f e}$ mede o sobrerrisco (de hospitalização ou óbito) em relação à população geral. Usando esse indicador, a comorbidade com maior sobrerrisco dentre as estudadas foi a diabetes mellitus (Tabela 2). O sobrerrisco de hospitalização é em média $5,39(5,34-5,43)$ vezes maior para adultos com diabetes do que a média geral da população adulta brasileira. Ao comparar as faixas etárias, o sobrerrisco das pessoas com diabetes é fortemente influenciado pela idade, variando entre $2,82(2,72-2,93)$ dentre os adultos menores de 40 anos e 6,87 (6,81-6,93) dentre aqueles acima de 60 anos (Tabela 2). Essa diferença é ainda mais drástica quando compara-se o sobrerrisco de óbito dentre as pessoas com diabetes. Aqueles com mais de 60 anos apresentam um sobrerrisco 10 vezes maior do que a média populacional. Pessoas com doença renal crônica tiveram um sobrerrisco 4,1 vezes maior de hospitalização e 6,9 vezes maior de ir a óbito, comparado com a população em geral. O efeito da idade no sobrerrisco é mais evidente para as doenças renais do que para a diabetes. Enquanto nos jovens adultos com doença renal crônica, o sobrerrisco é 1,3 vezes a média populacional, nos idosos, esse sobrerrisco aumentou 14 vezes. Pessoas com DCV mostraram um sobrerrisco, em média, 2,2 vezes a média populacional. Mais uma vez, a idade é um fator que aumenta o sobrerrisco, na medida em que aqueles com 40 até 59 anos têm um sobrerrisco 1,4 vezes a média geral, e aqueles com mais de 60 anos têm o sobrerrisco 3,5 vezes. A presença de asma, por sua vez, só se constituiu num sobrerrisco quando associada a idades maiores do que 60 anos (Tabela 2). 
Figura 1

Distribuição da incidência de hospitalizações e óbitos por 1.000 habitantes e sobrerrisco estimado com intervalos de 95\% de credibilidade (IC95\%) para hospitalização e óbito por síndrome respiratória aguda grave em decorrência da COVID-19 (SRAG-COVID) por faixa etária.

1a) Incidência de hospitalizações e óbitos

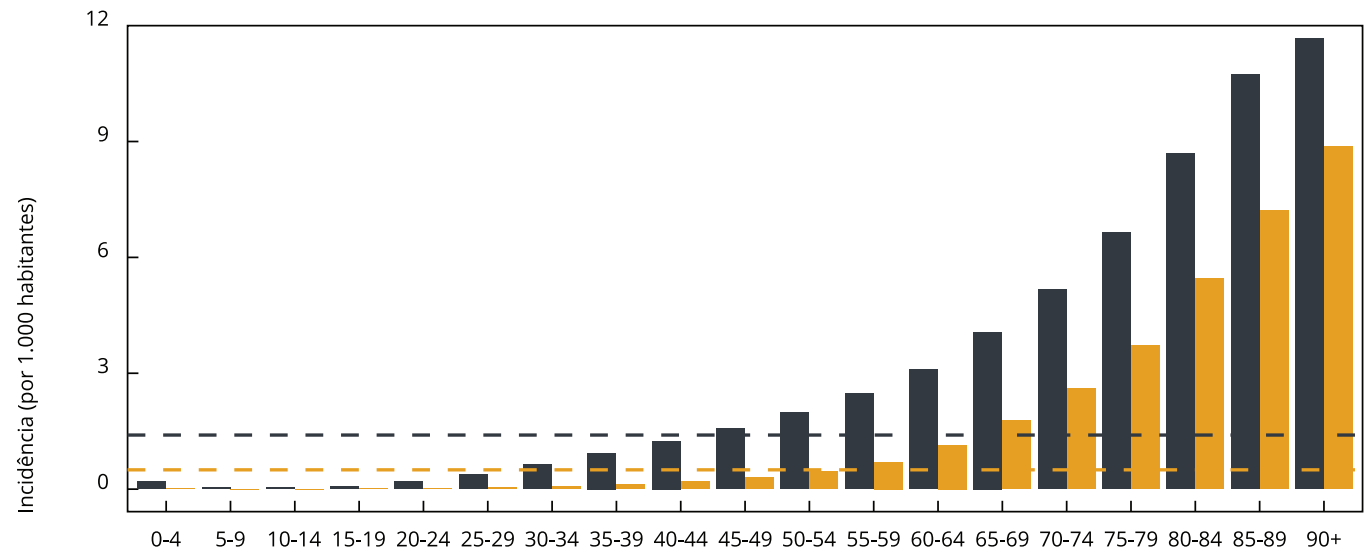

Hospitalizações

$\square$ Óbito

1b) Sobrerrisco estimado com IC95\%

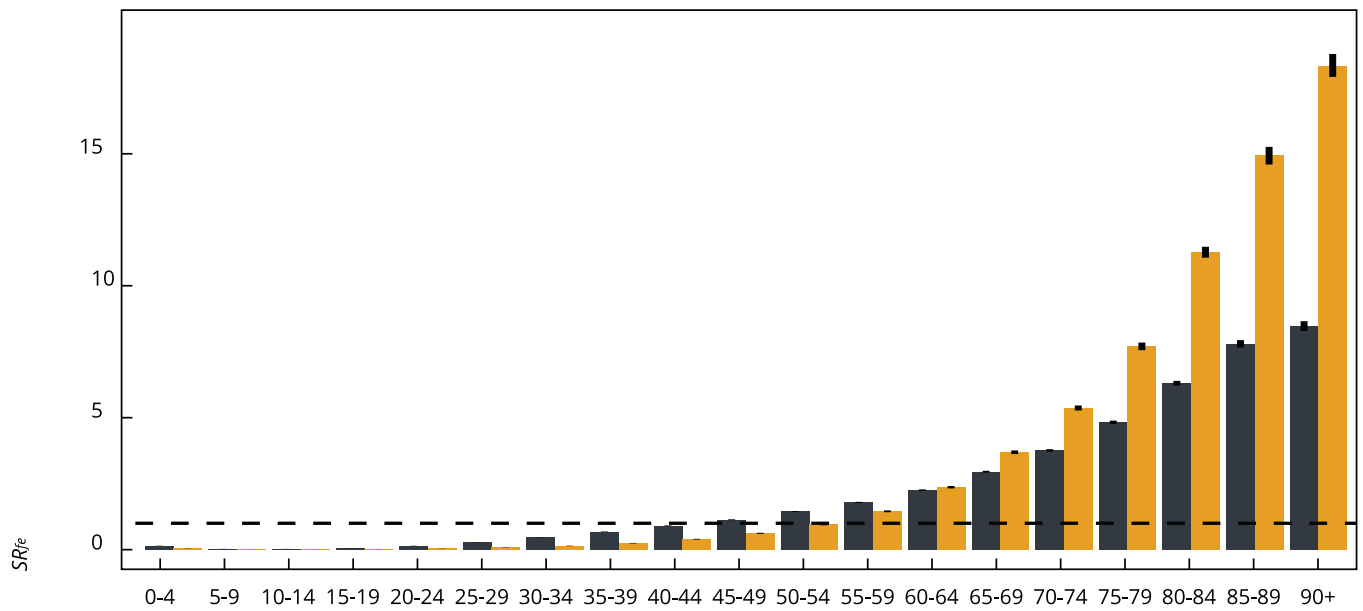

Hospitalizações

$\square$ Óbito

Notas: as linhas tracejadas em 1 a representam a incidência na população total para hospitalização $(1,4)$ e óbito $(0,5)$; a linha tracejada em 1 b representa $S R=1$, ou seja, ausência de sobrerrisco.

O segundo indicador apresentado na Tabela 2, $S R E_{c, f e}(f e)$, compara o sobrerrisco daqueles com comorbidade e certa faixa etária em relação à população total daquela faixa etária. Permite isolar o efeito da comorbidade do efeito já conhecido da idade. Dentre os adultos jovens, nota-se que a presença da comorbidade aumenta em muito o sobrerrisco, em especial, para diabetes [ $S R E_{c, f e}$ (18-39 anos) $=7,8]$, doença renal crônica $\left[S R E_{c, f e}(18-39\right.$ anos $\left.)=3,55\right]$ e DCV $\left[S R E_{c, f e}(18-39\right.$ anos $\left.)=1,75\right]$. Apenas asma não se mostrou um fator de sobrerrisco para os adultos jovens. Dentre os com 40-59 anos, ter diabetes está associado a um sobrerrisco de 3,0 em relação ao risco médio da população nessa faixa, e doença renal crônica tem um sobrerrisco de 1,96. Asma não se mostrou um fator de risco nessa faixa.

As $S R_{f e}$ para as faixas etárias utilizadas na Tabela 2 são: 0,360, 1,278 e 3,680 para hospitalização nas faixas etárias de 18-39, 40-59 e 60 anos ou mais, e 0,120, 0,807 e 5,431 para óbitos, respectivamente. 


\section{Tabela 1}

Prevalência de comorbidades na população de adultos do Brasil, segundo a Pesquisa Nacional de Saúde (PNS, 2013), total de casos estimados por comorbidade e faixa etária na população em 2020, números absolutos e taxas de incidência por mil habitantes de hospitalizações e óbitos por síndrome respiratória aguda grave em decorrência da COVID-19 (SRAG-COVID) até a 35ạ Semana Epidemiológica de 2020.

\begin{tabular}{|c|c|c|c|c|c|c|}
\hline \multirow[t]{2}{*}{ Faixa etária (anos) } & \multicolumn{2}{|c|}{ Estimativas para o Brasil (PNS) } & \multicolumn{4}{|c|}{ SRAG-COVID } \\
\hline & $\begin{array}{c}\text { Prevalência } \\
\text { (IC95\%) }\end{array}$ & Total de casos & Hospitalizações & $\begin{array}{c}\text { Hospitalizações } \\
\text { por } 1.000\end{array}$ & Óbitos & $\begin{array}{c}\text { Óbitos por } \\
1.000\end{array}$ \\
\hline \multicolumn{7}{|l|}{ Diabetes mellitus } \\
\hline $18-39$ & $1,0(0,8-1,2)$ & 752.295 & 2.922 & 3,9 & 688 & 0,9 \\
\hline $40-59$ & $7,3(6,6-7,9)$ & 3.879 .034 & 20.591 & 5,3 & 6.364 & 1,6 \\
\hline $60+$ & $18,1(16,9-19,3)$ & 5.301 .610 & 50.142 & 9,5 & 26.323 & 5,0 \\
\hline Total de adultos & - & 9.932 .938 & 73.655 & 7,4 & 33.375 & 3,4 \\
\hline \multicolumn{7}{|l|}{ Doença cardiovascular } \\
\hline $18-39$ & $7,2(6,3-8,1)$ & 5.416 .521 & 4.715 & 0,9 & 891 & 0,2 \\
\hline $40-59$ & $31,9(29,6-34,2)$ & 16.950 .846 & 32.858 & 1,9 & 8.667 & 0,5 \\
\hline $60+$ & $62,0(58,5-65,5)$ & 18.160 .210 & 86.312 & 4,8 & 44.539 & 2,5 \\
\hline Total de adultos & - & 40.527 .577 & 123.885 & 3,1 & 54.097 & 1,3 \\
\hline \multicolumn{7}{|l|}{ Doença renal crônica } \\
\hline $18-39$ & $0,6(0,5-0,8)$ & 451.377 & 796 & 1,8 & 249 & 0,6 \\
\hline $40-59$ & $1,8(1,5-2,1)$ & 956.474 & 3.304 & 3,5 & 1.500 & 1,6 \\
\hline $60+$ & $2,8(2,2-3,3)$ & 820.139 & 8.595 & 10,5 & 5.673 & 6,9 \\
\hline Total de adultos & - & 2.227 .989 & 12.695 & 5,7 & 7.422 & 3,3 \\
\hline \multicolumn{7}{|l|}{ Asma } \\
\hline 18-39 & $4,6(4,2-5,1)$ & 3.460 .555 & 1.497 & 0,4 & 172 & 0,0 \\
\hline $40-59$ & $3,9(3,5-4,3)$ & 2.072 .361 & 2.760 & 1,3 & 588 & 0,3 \\
\hline $60+$ & $4,8(4,1-5,5)$ & 1.405 .952 & 3.298 & 2,3 & 1.521 & 1,1 \\
\hline Total de adultos & - & 6.938 .867 & 7.555 & 1,1 & 2.281 & 0,3 \\
\hline \multicolumn{7}{|c|}{ Outra pneumopatia crônica } \\
\hline Total de adultos & $1,8(1,6-2,0)$ & 2.837 .836 & 11.015 & 3,9 & 6.129 & 2,2 \\
\hline
\end{tabular}

IC95\%: intervalo de 95\% de credibilidade.

\section{Discussão}

Neste artigo, nós identificamos grupos de risco para COVID-19 nos primeiros seis meses de pandemia no Brasil, a fim de auxiliar na definição de grupos prioritários para o Plano Nacional de Operacionalização da Vacinação contra a COVID-19 elaborado pelo PNI. Fatores como sexo masculino, faixas etárias mais altas e presença de comorbidades estão associados com sobrerrisco de hospitalização e óbito por SRAG-COVID, assim como encontrado em outros estudos 12,13,17,18.

O sexo masculino apresentou sobrerrisco para hospitalizações e óbitos de $33 \%$ e $41 \%$ maior do que o feminino, respectivamente. Apesar das proporções de casos entre os sexos não apresentarem diferenças significativas, homens têm mais chance de desenvolver casos graves e ir a óbito devido a diferenças biológicas, como mecanismos de resposta imunológica, assim como comportamentais e socioculturais relacionadas a consumo de álcool e tabaco, trabalho/ocupação e adesão a medidas de proteção 19,20 .

A idade é um forte e bem estabelecido fator de risco para hospitalização e óbito por COVID-19 12,21,22. Pertencer a faixas etárias mais jovens, até 40-44 anos, conferiu proteção relativa para hospitalização ou óbito durante os primeiros meses da pandemia, comparado com o risco médio geral. A partir de 45-49 anos há sobrerrisco, podendo alcançar valor oito vezes maior na faixa etária de 90 anos ou mais $\left(S R_{f e}=8,466\right)$. Em relação ao sobrerrisco de óbito, as faixas etárias mais jovens apresen- 
Tabela 2

$S R_{c, f e}$ e $S R E_{c, f e}(f e)$ para hospitalização e óbito por síndrome respiratória aguda grave em decorrência da COVID-19 (SRAG-COVID) e intervalos de $95 \%$ de credibilidade (IC95\%).

\begin{tabular}{|c|c|c|c|c|}
\hline \multirow{2}{*}{$\begin{array}{l}\text { Comorbidade/Faixa etária } \\
\text { (anos) }\end{array}$} & \multicolumn{2}{|c|}{ Hospitalização } & \multicolumn{2}{|c|}{ Óbito } \\
\hline & $\begin{array}{c}S R_{c, f e} \\
(I C 95 \%)\end{array}$ & $\begin{array}{c}S R E_{c, f e}(f e) \\
(I C 95 \%)\end{array}$ & $\begin{array}{c}S R_{c, f e} \\
(I C 95 \%)\end{array}$ & $\begin{array}{c}\operatorname{SRE}_{c, f e}(f e) \\
(I C 95 \%)\end{array}$ \\
\hline \multicolumn{5}{|l|}{ Diabetes mellitus } \\
\hline $18-39$ & $2,823(2,727-2,930)$ & $7,824(7,545-8,139)$ & $1,896(1,752-2,040)$ & $15,790(14,561-17,016)$ \\
\hline $40-59$ & $3,853(3,796-3,907)$ & $3,014(2,969-3,058)$ & 3,392 (3,309-3,483) & $4,202(4,085-4,312)$ \\
\hline $60+$ & $6,868(6,809-6,932)$ & $1,866(1,847-1,886)$ & $10,265(10,129-10,410)$ & $1,890(1,864-1,915)$ \\
\hline Total de adultos * & $5,385(5,342-5,429)$ & $4,185(4,152-4,219)$ & $6,949(6,864-7,038)$ & 5,190 5,129-5,255) \\
\hline \multicolumn{5}{|l|}{ Doença cardiovascular } \\
\hline $18-39$ & $0,632(0,615-0,650)$ & $1,754(1,702-1,806)$ & $0,340(0,319-0,360)$ & $2,837(2,624-3,044)$ \\
\hline $40-59$ & $1,408(1,393-1,423)$ & $1,101(1,087-1,115)$ & $1,057(1,036-1,081)$ & $1,310(1,276-1,343)$ \\
\hline $60+$ & $3,450(3,424-3,476)$ & $0,938(0,930-0,945)$ & $5,071(5,014-5,127)$ & $0,934(0,923-0,944)$ \\
\hline Total de adultos * & $2,220(2,206-2,236)$ & $1,725(1,714-1,737)$ & $2,760(2,732-2,789)$ & $2,062(2,042-2,083)$ \\
\hline \multicolumn{5}{|l|}{ Doença renal crônica } \\
\hline $18-39$ & $1,280(1,194-1,369)$ & $3,555(3,318-3,800)$ & $1,144(1,005-1,288)$ & $9,530(8,369-10,746)$ \\
\hline $40-59$ & $2,510(2,425-2,596)$ & $1,964(1,897-2,034)$ & $3,246(3,081-3,406)$ & $4,024(3,805-4,237)$ \\
\hline $60+$ & $7,609(7,449-7,762)$ & $2,066(2,020-2,109)$ & $14,295(13,942-14,687)$ & $2,634(2,569-2,702)$ \\
\hline Total de adultos * & $4,138(4,068-4,205)$ & $3,216(3,159-3,277)$ & $6,891(6,741-7,052)$ & $5,149(5,027-5,270)$ \\
\hline \multicolumn{5}{|l|}{ Asma } \\
\hline 18-39 & $0,315(0,299-0,330)$ & $0,871(0,827-0,915)$ & $0,104(0,089-0,119)$ & $0,861(0,739-1,004)$ \\
\hline $40-59$ & $0,967(0,931-1,007)$ & $0,757(0,729-0,785)$ & $0,588(0,538-0,635)$ & $0,729(0,671-0,789)$ \\
\hline $60+$ & $1,703(1,648-1,766)$ & $0,463(0,447-0,478)$ & $2,237(2,130-2,351)$ & $0,412(0,391-0,433)$ \\
\hline Total de adultos * & $0,790(0,773-0,808)$ & $0,615(0,601-0,628)$ & $0,680(0,651-0,708)$ & $0,508(0,487-0,529)$ \\
\hline \multicolumn{5}{|l|}{ Outra pneumopatia crônica } \\
\hline Total de adultos * & $2,818(2,764-2,868)$ & $2,190(2,150-2,230)$ & $4,465(4,356-4,576)$ & $3,338(3,259-3,421)$ \\
\hline
\end{tabular}

$S R_{c, f e}$ : cálculo do sobrerrisco associado a uma comorbidade e efeito conjunto da própria faixa etária; $S R E_{c, f e}(f e)$ : sobrerrisco associado a uma comorbidade específica, dentro de uma faixa etária específica.

* Nas linhas de totais de adultos, a $S R_{c, f e}$ usa como referência a taxa de incidência da população geral, enquanto a $S R E_{c, f e}(f e)$ usa, como referência, a taxa de incidência na população de adultos.

tam proteção relativa forte até 24 anos, com $S R_{f e}$ bem próximo de zero. De 55-59 anos há sobrerrisco aumentado, podendo ser quase 18 vezes maior na faixa etária de 90 anos ou mais. Importante pontuar que o crescimento não é exponencial quando se trata dos números absolutos e proporções de casos, uma vez que há concentração nas faixas etárias de 15-69 anos. Isso pode estar relacionado com a maior exposição dessas faixas etárias por constituírem a força de trabalho ativa e estarem sujeitas a uma maior tendência à flexibilização das medidas de distanciamento 21.

Ranzani et al. 22 mostraram que uma grande proporção, 84\%, dos pacientes hospitalizados com COVID-19 no Brasil apresentaram pelo menos uma comorbidade, porém, não há informações específicas para as comorbidades. Dentre as comorbidades avaliadas neste estudo, doença renal crônica, diabetes mellitus, outra pneumopatia crônica e DCV apresentaram maior risco de hospitalizações e óbitos. Ter doença renal crônica ou diabetes mellitus e idade acima de 60 anos mostrou-se um fator ainda mais forte, alcançando sobrerrisco de óbito 10 a 14 vezes maior do que a população geral. Outros estudos mostram essas doenças como importantes fatores de risco para infecção 18 , hospitalização 13, e óbito por COVID-19 23.

De forma geral, em todas as comorbidades há um sobrerrisco específico por faixa etária mais alto em idades menores, com um gradiente de diminuição em faixas mais altas, padrão que se inverte quando consideramos o sobrerrisco em relação à população geral, tanto para hospitalização quanto para óbito. Esse fenômeno ocorre porque ao compararmos com a população geral, o sobrerrisco é 
combinado com aquele específico da faixa etária, que, naturalmente, tem um impacto muito grande e é preponderante. Por exemplo, para as DCV não há evidência de sobrerrisco na população jovem, 18-39 anos, quando comparado com a população geral. No entanto, há um sobrerrisco de hospitalização em pessoas jovens com DCV 1,7 vezes maior do que em pessoas da mesma faixa etária. Para óbito, esse sobrerrisco é 2,8 vezes maior.

Para asma não há evidências de sobrerrisco, apesar de apresentar um sobrerrisco de hospitalização e óbito quando comparado à população geral em idades mais avançadas. Esse sobrerrisco é atribuído somente à idade, uma vez que o sobrerrisco de hospitalização (e óbito) em pessoas acima de 60 anos com asma é 1,7 (2,2) vezes maior do que na população geral, enquanto o sobrerrisco de hospitalização (e óbito) em pessoas acima de 60 anos é 3,7 (5,4) vezes maior. O efeito protetor relativo para asma foi relatado em outros trabalhos e algumas hipóteses têm sido discutidas como a pronta adoção de medidas de proteção 24; aspectos relacionados à resposta imune do tipo 2 que causam o acúmulo de eosinófilos 24,25,26,27; terapêutica comumente indicada para asma 28,29,30; e má descrição das comorbidades para COVID-19 24. No entanto, ainda são necessários estudos mais aprofundados para estabelecer as causas desse efeito protetor da asma para COVID-19.

A presente análise fez uso dos dados disponíveis no sistema de notificação SIVEP-Gripe (https:// sivepgripe.saude.gov.br/sivepgripe), aproveitando assim a infraestrutura e os fluxos já existentes na vigilância de síndromes respiratórias no país. A importância do SIVEP-Gripe durante a pandemia foi demonstrada claramente pelo acompanhamento feito por meio do sistema de monitoramento de casos de SRAG, o InfoGripe (http://info.gripe.fiocruz.br), que foi capaz de identificar o impacto do novo coronavírus na rede hospitalar antes mesmo da confirmação laboratorial dos primeiros casos 31 .

Esse estudo tem algumas limitações que são inerentes a dados coletados para fins de vigilância. Primeiramente, há um potencial viés de preenchimento das fichas de notificação e digitalização destas, sem acompanhamento direto caso a caso na rede hospitalar, como ocorreria num estudo clínico. Isso é exemplificado pelo não preenchimento entre os campos das 5 comorbidades analisadas neste artigo, que varia entre $8,8 \%$ em DCV e $25,9 \%$ em asma, sendo que estas se restringem àquelas listadas explicitamente como variáveis individuais no banco do SIVEP-Gripe. Por construção, os campos não preenchidos de comorbidades provavelmente indicam a ausência delas, contudo, é possível que existam casos de pessoas com comorbidades sem o registro adequado, o que implicaria em uma subestimação dos sobrerriscos calculados. Além disso, esses campos são preenchidos a partir das informações repassadas pelo(a) paciente ou responsável, podendo gerar subnotificação. No entanto, essa é a mesma abordagem utilizada na PNS, o que favorece a comparabilidade dos dados. Uma diferença importante - e que pode minimizar falhas quando se trata da PNS - é que o preenchimento é feito após padronização e treinamento para a realização das perguntas e/ou coleta dos dados pelos entrevistadores da pesquisa.

Apesar de termos assumido que as definições de morbidade da PNS e do SIVEP-Gripe são equivalentes, é possível que não sejam. Por exemplo, a primeira usa o termo insuficiência renal crônica, e a segunda, doença renal crônica. Como informado na seção de Métodos, DCV foi criada a partir de doença cardiovascular crônica e informações do campo aberto sobre hipertensão, mas é possível que isso não seja suficiente para compatibilização perfeita das variáveis, embora não acreditemos que eventuais diferenças sejam suficientes para invalidar as comparações.

Outra importante limitação é a subestimação da incerteza calculada para o sobrerrisco, uma vez que as populações são estimativas vindas de projeções do IBGE ou estimativas da PNS, e essas incertezas foram ignoradas no cálculo da distribuição do sobrerrisco. Outro potencial viés está relacionado aos dados da PNS utilizados nas análises. Esses dados são referentes ao ano de 2013 e, portanto, podem estar desatualizados para representar o estado de saúde com relação às morbidades da população geral no ano de 2020 .

O presente estudo é uma contribuição para a elaboração do plano nacional de vacinação, mas não deve ser considerado isoladamente, pois existem outros fatores de risco que não foram considerados por não constarem nas bases de dados disponíveis (em pelo menos uma das bases utilizadas nas análises), como, por exemplo, cirrose hepática 32, doença falciforme 33, síndrome de Down $34 \mathrm{e}$ câncer 35,36. Existem evidências também de maior morbidade e mortalidade por doenças de transmissão respiratória em populações como a de indígenas 37 e de privados de liberdade 38 . Outros estudos de natureza quantitativa ou qualitativa são necessários para complementar a definição dos grupos de 
risco. Além do risco individual, outros elementos devem nortear a definição de grupos prioritários. A vacina contra COVID-19 é um bem comum e seu uso deve ser guiado por valores de equidade e promoção da saúde. Sua distribuição, especialmente num contexto de limitação de doses, deve considerar princípios de maximização de bem-estar humano (redução de mortalidade e sofrimento), reciprocidade com aqueles que mais se dedicam à frente de batalha e cura (profissionais de saúde), equidade (acesso dos mais vulneráveis) e legitimidade 9 . Esses objetivos só poderão ser alcançados através de uma pactuação com a sociedade.

Além disso, a epidemia de COVID-19 tem efeitos diretos e indiretos nos estratos populacionais com comorbidades. O efeito direto é o aumento de risco para hospitalizações e óbitos em casos de SRAG-COVID com presença de comorbidades, já bem documentado. O efeito indireto é a redução da assistência e cuidado dessas condições, por causa da redução de mobilidade e acesso aos serviços de saúde, o que pode ter resultado no descontrole dessas condições. Faz-se, dessa forma, necessário ressaltar o impacto da COVID-19 em doenças não transmissíveis. A retomada de políticas públicas de promoção da saúde para estas, inclusive como uma forma de resposta à pandemia e complementar à vacina, deve ser incluída como parte urgente e essencial para minimizar casos graves e mortes decorrentes da associação entre doença e condição de base ${ }^{39}$. Em todo o mundo, recursos humanos e financeiros foram direcionados para o enfrentamento da pandemia, o que também impactou no acompanhamento dessas doenças ${ }^{39}$, que, por exemplo, é realizado pelas equipes de saúde da família do SUS no Brasil.

No início de 2020, quando a pandemia de COVID-19 foi declarada, a seguinte pergunta foi posta: estamos preparados para enfrentar essa pandemia no Brasil? ${ }^{40}$. O subfinanciamento e o desmonte do SUS têm causado uma redução enorme de recursos humanos e de infraestrutura que impactam diretamente na qualidade dos dados e, certamente, no sucesso do PNI em alcançar a cobertura vacinal necessária.

Apesar das limitações expostas, o presente estudo foi capaz de dar subsídio ao Plano Nacional de Operacionalização da Vacinação contra a COVID-19 do PNI através da identificação de grupos populacionais com sobrerrisco de hospitalização e óbito pela doença. Foram utilizados dados que são coletados na rotina dos estabelecimentos de saúde, ressaltando a importância da disponibilização de dados de qualidade. A pandemia demonstrou claramente a realidade vigente no país, que tem sido apontada há anos por pesquisadores e trabalhadores da saúde: a necessidade de se ter um sistema de notificação bem operado e preenchido por pessoas devidamente treinadas e a urgência em garantir investimentos para o SUS 40. 


\section{Colaboradores}

R. M. Lana contribuiu na concepção do estudo, processamento de dados, análise, redação e revisão crítica do texto. L. P. Freitas, C. T. Codeço e A. G. Pacheco contribuíram na interpretação dos dados, redação e revisão crítica do texto. L. M. F. Carvalho contribuiu na análises, interpretação dos dados e revisão crítica do texto. D. A. M. Villela, F. C. Coelho, V. B. G. Porto e C. Gava contribuíram na interpretação dos dados e revisão crítica do texto. O. G. Cruz contribuiu na interpretação dos dados. R. P. Niquini contribuiu na concepção do estudo e interpretação dos dados. M. F. C. Gomes contribuiu na concepção do estudo, coleta e processamento de dados, redação e revisão crítica do texto. L. S. Bastos contribuiu na concepção do estudo, coleta e processamento de dados, análise, redação e revisão crítica do texto. Todos os autores revisaram e aprovaram a versão final para publicação.

\section{Informações adicionais}

ORCID: Raquel Martins Lana (0000-0002-75731364); Laís Picinini Freitas (0000-0001-90129382); Cláudia Torres Codeço (0000-0003-1174178X); Antônio Guilherme Pacheco (0000-00033095-1774); Luiz Max Fagundes de Carvalho (0000-0001-5736-5578); Daniel Antunes Maciel Villela (0000-0001-8371-2959); Flávio Codeço Coelho (0000-0003-3868-4391); Oswaldo Gonçalves Cruz (0000-0002-3289-3195); Roberta Pereira Niquini (0000-0003-1075-3113); Victor Bertollo Gomes Porto (0000-0002-3112-6154); Caroline Gava (0000-0001-6287-1715); Marcelo Ferreira da Costa Gomes (0000-0003-4693-5402); Leonardo Soares Bastos (0000-0002-1406-0122).

\section{Agradecimentos}

À Rede Nacional de Vigilância de Influenza (LACENs, NICs, vigilâncias estaduais e municipais, e GT-Influenza, Departamento de Imunização e Doenças Transmissíveis da Secretaria de Vigilância em Saúde do Ministério da Saúde) pela parceria. Ao Inova Fiocruz, Conselho Nacional de Desenvolvimento Científico e Tecnológico (CNPq - ref. 441057/2020-9, ref. 309569/20192 e 307489/2018-3), Fundação de Amparo à Pesquisa do Estado do Rio de Janeiro (FAPERJ E26/203.172/2) pelo financiamento.

\section{Referências}

1. Candido DS, Claro IM, Jesus JG, Souza WM, Moreira FRR, Dellicour S, et al. Evolution and epidemic spread of SARS-CoV-2 in Brazil. Science 2020; 369:1255-60.

2. Coelho FC, Lana RM, Cruz OG, Codeco CT, Villela D, Bastos LS, et al. Assessing the potential impact of COVID-19 in Brazil: mobility, morbidity and the burden on the Health Care System. medRxiv 2020; 26 mar. http://medrxiv.org/lookup/doi/10.1101/2020 $.03 .19 .20039131 \mathrm{v} 2$.

3. Secretaria de Vigilância em Saúde, Ministério da Saúde. Guia de vigilância epidemiológica emergência de saúde pública de importância nacional pela doença pelo coronavírus 2019: vigilância de síndromes respiratórias agudas COVID-19. Brasília: Ministério da Saúde; 2020.

4. Domingues CMAS. Desafios para a realização da campanha de vacinação contra a COVID-19 no Brasil. Cad Saúde Pública 2021; 37:e00344620.

5. World Health Organization. Draft landscape and tracker of COVID-19 candidate vaccines. https://www.who.int/publications/m/item/ draft-landscape-of-covid-19-candidate-vac cines (acessado em 24/Fev/2021).

6. World Health Organization. Coronavirus disease (COVID-19): vaccines. https://www. who.int/news-room/q-a-detail/coronavirusdisease-(covid-19)-vaccines (acessado em 27/ Jan/2021).

7. Agência Nacional de Vigilância Sanitária. Uso emergencial: confira votos, relatório e apresentações da reunião. https://www.gov.br/ anvisa/pt-br/assuntos/noticias-anvisa/2021/ confira-materiais-da-reuniao-extraordinariada-dicol (acessado em 16/Fev/2021).

8. Vacina da Pfizer é a 1a contra a Covid a obter registro definitivo no Brasil. G1 2021; 23 fev. https://g1.globo.com/bemestar/vacina/noti cia/2021/02/23/anvisa-concede-registro-de finitivo-a-vacina-da-pfizer.ghtml.

9. World Health Organization. Coronavirus disease (COVID-19): vaccine access and allocation. https://www.who.int/news-room/qa-detail/coronavirus-disease-(covid-19)-vac cine-access-and-allocation (acessado em 16/ Fev/2021).

10. Ministério da Saúde. Plano Nacional de operacionalização da vacinação contra a COVID-19. https://www.gov.br/saude/pt-br/ media/pdf/2020/dezembro/16/plano_vacina cao_versao_eletronica-1.pdf (acessado em 15/ Jan/2021).

11. Niquini RP, Lana RM, Pacheco AG, Cruz OG, Coelho FC, Carvalho LM, et al. SRAG por COVID-19 no Brasil: descrição e comparação de características demográficas e comorbidades com SRAG por influenza e com a população geral. Cad Saúde Pública 2020; 36:e00149420. 
12. Andrade CLT, Pereira CCA, Martins M, Lima SML, Portela MC. COVID-19 hospitalizations in Brazil's Unified Health System (SUS). PLoS One 2020; 15:e0243126.

13. Petrilli CM, Jones SA, Yang J, Rajagopalan H, O'Donnell L, Chernyak Y, et al. Factors associated with hospital admission and critical illness among 5279 people with coronavirus disease 2019 in New York City: prospective cohort study. BMJ 2020; 369:m1966.

14. Instituto Brasileiro de Geografia e Estatística. Projeção da população do Brasil e Unidades da Federação por sexo e idade para o período 2000-2030. https://datasus.saude.gov. br/populacao-residente/ (acessado em 17/ Out/2020)

15. Instituto Brasileiro de Geografia e Estatística. Pesquisa Nacional de Saúde 2013. Percepção do estado de saúde, estilos de vida e doenças crônicas. https://www.ibge.gov.br/estatisti cas/sociais/saude/9160-pesquisa-nacional-desaude.html (acessado em 17/Out/2020).

16. Malta DC, Stopa SR, Szwarcwald CL, Gomes NL, Silva Júnior JB, Reis AAC. A vigilância e o monitoramento das principais doenças crônicas não transmissíveis no Brasil - Pesquisa Nacional de Saúde, 2013. Rev Bras Epidemiol 2015; 18:3-16.

17. Ortiz-Prado E, Simbaña-Rivera K, Barreno LG, Diaz AM, Barreto A, Moyano C, et al. Epidemiological, socio-demographic and clinical features of the early phase of the COVID-19 epidemic in Ecuador. PLoS Negl Trop Dis 2021; 15:e0008958.

18. Harrison SL, Fazio-Eynullayeva E, Lane DA, Underhill P, Lip GYH. Comorbidities associated with mortality in 31,461 adults with COVID-19 in the United States: a federated electronic medical record analysis. PLoS Med 2020; 17:e1003321.

19. Peckham H, Gruijter NM, Raine C, Radziszewska A, Ciurtin C, Wedderburn LR, et al. Male sex identified by global COVID-19 meta-analysis as a risk factor for death and ITU admission. Nat Commun 2020; 11:6317.

20. Abate BB, Kassie AM, Kassaw MW, Aragie TG, Masresha SA. Sex difference in coronavirus disease (COVID-19): a systematic review and meta-analysis. BMJ Open 2020; 10:e040129.

21. Xiong D, Zhang L, Watson GL, Sundin P, Bufford T, Zoller JA, et al. Pseudo-likelihood based logistic regression for estimating COVID-19 infection and case fatality rates by gender, race, and age in California. Epidemics 2020; 33:100418.

22. Ranzani OT, Bastos LSL, Gelli JGM, Marchesi JF, Baião F, Hamacher S, et al. Characterisation of the first 250000 hospital admissions for COVID-19 in Brazil: a retrospective analysis of nationwide data. Lancet Respir Med 2021; 9:407-18.
23. Ssentongo P, Ssentongo AE, Heilbrunn ES, Ba DM, Chinchilli VM. Association of cardiovascular disease and 10 other pre-existing comorbidities with COVID-19 mortality: a systematic review and meta-analysis. PLoS One 2020; 15:e0238215.

24. Mendes NF, Jara CP, Mansour E, Araújo EP, Velloso LA. Asthma and COVID-19: a systematic review. Allergy Asthma Clin Immunol 2021; 17:5.

25. Wakabayashi M, Pawankar R, Narazaki H, Ueda T, Itabashi T. Coronavirus disease 2019 and asthma, allergic rhinitis: molecular mechanisms and host-environmental interactions. Curr Opin Allergy Clin Immunol 2021; 21:1-7.

26. Lee SC, Son KJ, Han CH, Jung JY, Park SC. Impact of comorbid asthma on severity of coronavirus disease (COVID-19). Sci Rep 2020; 10:21805.

27. Carli G, Cecchi L, Stebbing J, Parronchi P, Farsi A. Is asthma protective against COVID-19? Allergy 2020. [Epub ahead of print].

28. Kumar K, Hinks TSC, Singanayagam A. Treatment of COVID-19-exacerbated asthma: should systemic corticosteroids be used? Am J Physiol Lung Cell Mol Physiol 2020. 318:L1244-L1247.

29. Morais-Almeida M, Bousquet J. COVID-19 and asthma: to have or not to have T2 inflammation makes a difference? Pulmonology 2020; 26:261-3.

30. Global Initiative for Asthma. Recommendations for inhaled asthma controller medications. https://ginasthma.org/recommenda tions-for-inhaled-asthma-controller-medica tions/ (acessado em 23/Fev/2021).

31. Bastos LS, Niquini RP, Lana RM, Villela DAM, Cruz OG, Coelho FC, et al. COVID-19 e hospitalizações por SRAG no Brasil: uma comparação até a 12a semana epidemiológica de 2020. Cad Saúde Pública 2020; 36:e00070120.

32. Ioannou GN, Liang PS, Locke E, Green P, Berry K, O'Hare AM, et al. Cirrhosis and SARSCoV-2 infection in US Veterans: risk of infection, hospitalization, ventilation and mortality. Hepatology 2021; 74:322-35.

33. Centers for Disease Control and Prevention. Evidence used to update the list of underlying medical conditions that increase a person's risk of severe illness from COVID-19. https:// www.cdc.gov/coronavirus/2019-ncov/needextra-precautions/evidence-table.html (acessado em 24/Fev/2021).

34. Clift AK, Coupland CAC, Keogh RH, Hemingway H, Hippisley-Cox J. COVID-19 mortality risk in down syndrome: results from a cohort study of 8 million adults. Ann Intern Med 2021; 174:572-6. 
35. Ferrari BL, Ferreira CG, Menezes M, De Marchi P, Canedo J, Melo AC, et al. Determinants of COVID-19 mortality in patients with cancer from a community oncology practice in Brazil. JCO Glob Oncol 2021; 7:46-55.

36. Carreira H, Strongman H, Peppa M, McDonald HI, dos-Santos-Silva I, Stanway S, et al. Prevalence of COVID-19-related risk factors and risk of severe influenza outcomes in cancer survivors: a matched cohort study using linked English electronic health records data. Research Paper 2020; 29:100656.

37. Cardoso AM, Resende PC, Paixao ES, Tavares FG, Farias YN, Barreto CTG, et al. Investigation of an outbreak of acute respiratory disease in an indigenous village in Brazil: Contribution of Influenza A(H1N1)pdm09 and human respiratory syncytial viruses. PLoS One 2019; 14:e0218925.
38. Sacchi FPC, Praça RM, Tatara MB, Simonsen V, Ferrazoli L, Croda MG, et al. Prisons as Reservoir for Community Transmission of Tuberculosis, Brazil. Emerg Infect Dis 2015; 21:452-5.

39. World Health Organization. The impact of the COVID-19 pandemic on noncommunicable disease resources and services: results of a rapid assessment. https://apps.who.int/ iris/handle/10665/334136 (acessado em 24/ Fev/2021).

40. Lana RM, Coelho FC, Gomes MFC, Cruz OG, Bastos LS, Villela DAM, et al. Emergência do novo coronavírus (SARS-CoV-2) e o papel de uma vigilância nacional em saúde oportuna e efetiva. Cad Saúde Pública 2020; 36:e00019620. 


\section{Abstract}

In a context of community transmission and shortage of vaccines, COVID-19 vaccination should focus on directly reducing the morbidity and mortality caused by the disease. It is thus essential to define priority groups for vaccination by the National Immunization Program (PNI in Portuguese), based on the risk of hospitalization and death from the disease. We thus calculated overrisk according to sex, age group, and comorbidities using hospitalization and death records from severe acute respiratory illness with confirmation of COVID-19 (SARI-COVID) in all of Brazil in the first six months of the epidemic. Higher overrisk was associated with male sex (hospitalization = 1.1 and death $=1.2$ ), age over 45 years for hospitalization $\left(\mathrm{OvR}_{\mathrm{ag}}\right.$ ranging from 1.1 to 8.5), and age over 55 year for death $\left(\mathrm{OvR}_{\mathrm{ag}}\right.$ ranging from 1.5 to 18.3). In the groups of comorbidities, chronic kidney disease, diabetes mellitus, cardiovascular disease, and chronic lung disease were associated with overrisk, while there was no such evidence for asthma. Chronic kidney disease or diabetes plus age over 60 showed an even stronger association, reaching overrisk of death 14 and 10 times greater than in the general population, respectively. For all the comorbidities, there was higher overrisk at older ages, with a downward gradient in the oldest age groups. This pattern was reversed when examining overrisk in the general population, for both hospitalization and death. The current study provided evidence of overrisk of hospitalization and death from SARI-COVID, assisting the definition of priority groups for COVID-19vaccination.

COVID-19; Severe Acute Respiratory Syndrome; Epidemiological Monitoring; Comorbidity

\section{Resumen}

En un contexto de transmisión comunitaria y escasez de vacunas, la vacunación contra la COVID-19 debe enfocarse en la reducción directa de la morbilidad y de la mortalidad causadas por la enfermedad. Por lo tanto, es fundamental la definición de grupos prioritarios para la vacunación por el Programa Nacional de Inmunizaciones (PNI), basada en el riesgo de hospitalización y óbito por la enfermedad. Para tal fin, calculamos el sobrerriesgo por sexo, franja de edad y comorbilidades mediante los registros de hospitalización $y$ óbito por sindrome respiratorio agudo grave con confirmación de COVID-19 (SRAG-COVID) en todo Brasil, durante los primeros seis meses de epidemia. Presentaron mayor sobrerriesgo personas del sexo masculino (hospitalización $=1,1$ y óbito $=1,2$ ), personas por encima de 45 años para hospitalización $\left(\mathrm{SR}_{\mathrm{fe}}\right.$ variando de 1,1 a 8,5) y personas por encima de 55 años para óbitos $\left(\mathrm{SR}_{\mathrm{fe}}\right.$ variando de 1,5 a 18,3). En los grupos de comorbilidades, enfermedad renal crónica, diabetes mellitus, enfermedad cardiovascular y neumopatía crónica ofrecieron sobrerriesgo, mientras que para el asma no hubo evidencia. Sufrir una enfermedad renal crónica o diabetes mellitus y tener 60 años o más mostró un factor todavía más fuerte, alcanzando sobrerriesgo de enfermedad 14 y 10 veces mayor que en la población general, respectivamente. Para todas las comorbilidades, hubo un sobrerriesgo más alto en edades mayores, con un gradiente de disminución en franjas más altas. Este patrón se invirtió cuando consideramos el sobrerriesgo en relación con la población general, tanto para hospitalización como para óbito. El presente estudio proporcionó evidencias respecto al sobrerriesgo de hospitalización y óbito por SRAG-COVID, ayudando en la definición de grupos prioritarios para la vacunación contra la COVID-19.

COVID-19; Síndrome Respiratorio Agudo Grave; Monitoreo Epidemiológico; Comorbilidad
Recebido em 24/Fev/2021

Versão final reapresentada em 21/Mai/2021 Aprovado em 29/Jul/2021 